https://doi.org/10.7203/Normas.2.4660

\title{
DE ERRORES Y ERRATAS. CÓMO CORREGIR Y NORMALIZAR UN TEXTO ACADÉMICO*
}

\author{
ABOUT ERRORS AND ERRATA. HOW TO CORRECT AND TO STANDARDIZE \\ AN ACADEMIC TEXT
}

Andrea ESTRADA

Universidad de Buenos Aires

Secrit-Conicet

\section{RESUMEN:}

En el presente artículo, se intenta demostrar en primer lugar, a partir de ejemplos de una revista académica de lingüística, que errores y erratas tienen el mismo protagonismo en la corrección de textos académicos. Pero además, que los criterios y procedimientos de normalización definida como el conjunto de decisiones y de procedimientos que tienden a unificar el estilo de un escrito en todos sus niveles- son fundamentales para que un conjunto de artículos científicos de autores varios o una serie de trabajos de un mismo autor, de distinto tema o etapas académicas, puedan llegar a convertirse en una publicación periódica o en un libro.

PALABRAS CLAVE: corrección de estilo, normalización, errores, erratas, discurso académico.

\section{ABSTRACT:}

In the present article, we first intend to demonstrate, by showing examples taken from an academic journal on Linguistics, that errors and errata are equally essential in correction of academic texts. In addition, the aim of our work is to show that both criteria and procedures towards normalization - this, defined as a set of decisions and procedures which tend to unify the style of a writing in all its levels - are the basis on which a group of scientific articles belonging to different authors, or else a body of work written by the same author, dealing with different topics or belonging to several academic stages, may become a periodical publication or a book.

KEY WORDS: style correction, normalization, errors, errata, academic discourse.

* Una primera versión de este artículo fue presentada en el Primer Congreso Internacional de Correctores en Lengua Española «Unidos por el mismo idioma», Buenos Aires, Aula Magna de la Facultad de Derecho, Universidad de Buenos Aires, 14 al 16 de septiembre de 2011. 


\section{INTRODUCCIÓN}

El título de este artículo, al aludir a los errores y a las erratas, intenta poner de manifiesto no solo la especificidad que entrañan los distintos tipos de corrección, es decir, la corrección de estilo y la corrección de pruebas, sino también el rol de los correctores en las diversas intervenciones a las que se ve sometido un texto científicoacadémico -artículo científico, ponencia de congreso, reseña crítica o avance de investigación- desde el original de autor hasta su publicación.

Ken Hyland (2000) define la escritura académica como el espacio interactivo y cognitivo en el cual los especialistas entablan un diálogo experto y conforman desde sus disciplinas intercambios discursivos con los cuales intentan que sus aportes sean reconocidos y aceptados por sus colegas. Como en cualquier publicación, entonces, también en la edición técnica, la corrección de errores y de erratas resulta de primordial importancia.

Pero comencemos por hacer un poco de historia. Porque, desde la perspectiva metodológica de la crítica textual o ecdótica, tal como señala José Luis Moure (2006:14), el error se define como «un desvío de la norma lingüística previamente plasmada en una gramática prescriptiva y una ortografía», pero que deviene en instrumento de identificación de fenómenos de habla. En efecto, esta disciplina se ocupa de relevar errores para estudiar la evolución de una lengua en aquellas etapas en la que no existía posibilidad de transcripción fonética, magnetofónica o electrónica. En el marco de los estudios filológicos, entonces, el error se transforma en un medio para aproximarse al habla real de un determinado momento histórico y para establecer cambios lingüísticos en marcha. Es por esa razón por la que, tal como señala José Luis Moure, la crítica textual «redimió parcialmente a los errores de su lastre negativo» en la medida en que hizo de ellos una condición necesaria para, por ejemplo, reconstruir una versión única y confiable de un manuscrito, a partir de los yerros reproducidos a lo largo del tiempo por distintos copistas.

Por el contrario, desde una perspectiva sincrónica y prescriptiva de la lengua, el error es considerado como un desvío de la norma vigente que debe ser erradicado porque puede condenar un texto a la ininteligibilidad.

Pero una cosa es el error y otra, la errata. Y para establecer sus diferencias debemos remontarnos a las dos etapas en las que la aparición de la imprenta dividió nuestra tradición escrituraria: por un lado, la manuscrita antigua o medieval en la que el filólogo-editor se enfrentaba con el error surgido por la intervención de numerosos copistas y, por el otro, la errata, una nueva dimensión del error que la imprenta se encargó de multiplicar y extender en el espacio y en el tiempo y que José Luis Moure (2006: 17) define, no sin un dejo de ironía, como «deslices poligenéticos que fatalmente persiguieron también a los operarios» (Moure, 2006: 17). 


\subsection{La corrección de estilo}

Pero volvamos ahora a la actualidad, en la cual la corrección ha adquirido una especificidad diferente a la de los tiempos imprenteriles. Las erratas son, en nuestros días, los errores que devienen de la composición digital, mientras que se reserva el término «error» a los de contenido, que se corrigen en la corrección de concepto o a los ortotipográficos, gramaticales o textuales (García Negroni y Estrada, 2006) de los que se ocupa la corrección de estilo.

Pero ¿qué es el estilo? Según Alicia Zorrilla (2004: 117), «el sintagma corrección de estilo confunde, sobremanera, a no pocos correctores y a los que no lo son, pues el estilo no se corrige». Para esta autora, la opción de llamar a la corrección revisión de originales «no describe con justeza esa labor».

Por su parte, para Mijaíl Bajtín, el estilo verbal de un enunciado es «la selección de los recursos léxicos, fraseológicos y gramaticales de la lengua», pero que, en la esfera del uso del lenguaje y en combinación con el contenido temático y la composición o estructuración de un texto, conforma el género discursivo. Dicho de otro modo, todo estilo está indisolublemente vinculado a las formas típicas de enunciados, de allí la frase de Mijaíl Bajtín (2002: 251) «Donde existe un estilo, existe un género». Es decir, cada autor confiere a su escritura un estilo propio e individual, a partir del conjunto de elecciones adoptadas según el tipo de texto o de práctica institucional en el que su contribución se inscriba.

Y a esta altura, surge un nuevo interrogante: ¿qué significa corregir el estilo? Porque no es lo mismo aplicar un principio normativo $-i$. e. corregir lo prescripto por la norma vigente- que seleccionar una opción estilística de acuerdo con criterios personales. En un caso, se trata de enmendar un error, mientras que en el otro, prima el criterio de selección entre varias posibilidades lingüísticas correctas.

Pero tal como revela Mijaíl Bajtín, no todos los géneros se prestan para reflejar un estilo individual de la misma manera; por ejemplo, en los géneros literarios, el estilo de cada autor forma parte de la intención del enunciado y, entonces, la corrección de textos de ficción resulta un tipo de corrección específica. En palabras de Mariana Bozetti (2010: 5):

[...] el corrector literario es un equilibrista, que debe medir con exactitud dónde apoyar el pie para no caerse, es decir, cuándo intervenir y cuándo abstenerse de hacerlo, porque no debe modificar aquellas elecciones que son propias del autor y que lo singularizan. Lo complicado es establecer en dónde radican. 
Por el contrario, en los géneros académicos, que se construyen a partir de ciertos rasgos estilísticos estandarizados, el estilo es un producto complementario, al decir de Mijaíl Bajtín, un «epifenómeno» del enunciado.

En síntesis, en la instancia de corrección de estilo de un texto académico, no solo se enmiendan errores, sino que $-\mathrm{y}$ es este el punto que me interesa destacar- se normaliza el estilo, es decir, se unifican criterios, tanto a nivel textual (tono, estructuración de las oraciones, tipo de enunciador e, incluso, la organización de la información) como paratextual (títulos, subtítulos, notas al pie, bibliografía, etc.). Esta intervención resulta fundamental para lograr que los rasgos estilísticos de los que habla Mijaíl Bajtín, respondan a la estandarización propia del género académico, definido de la siguiente manera por Silvia Senz (2006: 80-95):

[...] se denomina estilo científico o académico no solo a los aspectos específicos del lenguaje científico o académico y a los cánones compositivos y estructurales propios de una tipología discursiva científica o académica concreta, sino a todo el conjunto de estándares gráficos y terminológicos particulares de una determinada área de conocimiento, y a las normas de elaboración del trabajo científico y académico (deontología, reglas de trabajo documental y de presentación de materiales), comúnmente aceptados por la comunidad científica y académica que los desarrolla.

\subsection{Corrección de pruebas}

Pero veamos ahora qué sucede con la corrección de las primeras pruebas, antiguamente llamadas galeradas (texto sin paginar), y de las pruebas de página o segundas pruebas (texto paginado). Como señalé antes, en la etapa de corrección de pruebas, se enmiendan los errores emanados del proceso mismo de la composición -en la actualidad no ya de la imprenta, sino del plantado del texto por medios digitales (Quark o In design)-. Y, aunque las posibilidades de intervención sobre el texto están de alguna manera acotadas - de hecho, el original ya ha sido plantado y, por lo tanto, los cambios no pueden alterar el diseño ni la paginación sin provocar una significativa pérdida de tiempo y de trabajo-, no es cierto que para corregir pruebas no se necesiten también conocimientos específicos.

En síntesis, tanto la corrección de estilo como la de pruebas son dos prácticas necesarias y complementarias en la edición técnica, a pesar de que en la actualidad, se ha tendido a sobreestimar injustamente la corrección de estilo en detrimento de la de pruebas, por presuponer que la primera exige que el corrector posea rigurosas competencias -enciclopédicas, linguiísticas y normativas- (García Negroni y Estrada, 2006), mientras que para la corrección de pruebas, parecería suficiente contar con buena vista y un alto grado de concentración. A tal punto prevalece esta representación 
dicotómica difícil vs. fácil o complejo vs. mecánico en las dos instancias de la corrección, que suele decirse que el estilo «se corrige» y las pruebas «se revisan».

De alguna manera, creo que la corrección de pruebas tiene mala prensa porque equivocadamente es considerada algo mecánico, unívoco, basado tan solo en criterios tipográficos y visuales que, al dejar escaso margen a la creatividad, no requiere de competencias específicas. Quizás sea esta la razón además de por los consabidos vaivenes presupuestarios, por la cual la mayoría de las revistas académicas encargan la revisión de las pruebas a los propios autores.

\section{PROCEDIMIENTOS DE NORMALIZACIÓN EDITORIAL}

Pero vamos a detenernos, ahora, específicamente en un aspecto fundamental de la edición académica, la normalización, definida como el conjunto de decisiones y procedimientos que tienden a unificar el estilo de un escrito en todos sus niveles. Porque es mediante los procedimientos de normalización editorial que un conjunto de artículos científicos de autores varios puede llegar a conformar una publicación periódica; o una serie de trabajos de un mismo autor de distintas etapas académicas o de diferentes temas, puede convertirse en un libro si, tal como señala Patricia Piccolini (2006: 61), constituyen un «envase común», una obra en la cual el valor individual de cada uno de los textos borre sus diferencias y se fusione en el conjunto.

\subsection{La normalización de errores}

Los procedimientos de normalización, entonces, tienden a unificar las elecciones estilísticas de los escritores en relación con el género, en este caso el académico, y también en relación con el registro, es decir, con el contexto situacional en el cual se interrelacionan el tema de la comunicación (campo), la relación entre los participantes (tenor) y el papel del lenguaje en esa interacción (modo) (Reyes, 1998: 48-49).

Los rasgos lingüísticos asociados al campo son el vocabulario, las figuras, las alusiones culturales, el lenguaje especializado, etc.; al tenor: las formas de tratamiento, los apelativos, el uso de expresiones dialectales, el cambio de código, etc.; y al modo: las referencias a la situación, el uso del lenguaje oral, las jergas, el uso de expresiones dialectales, etc.

Tal como señala Graciela Reyes (1998: 49-50) el registro es la «asociación entre rasgos contextuales y sistemas linguísticos correspondientes» (op. cit: 49), de allí que una corrección que busque normalizar el estilo deberá concentrarse en enmendar 
aquellas elecciones estilísticas que no son absolutamente libres porque están limitadas por la situación comunicativa. Dicho de otro modo, normalizar implica corregir errores y erratas, pero también aquellos rasgos lingüísticos facultativos en los cuales el escritor de texto científico goza de un cierto grado de libertad de elección ${ }^{\dagger}$.

En lo que sigue, entonces, voy a presentar ejemplos extraídos de artículos científicos de lingüística pertenecientes a la revista académica Páginas de Guarda ${ }^{\ddagger}$ con la finalidad de mostrar los procedimientos de normalización en la corrección de estilo y en la de pruebas; pero además, para desterrar el preconcepto «creativo versus mecánico» con el que, como señalé antes, se las suele identificar, a pesar de que en ambas conviven los saberes (competencias propias del corrector de estilo) y las prácticas (técnicas de corrección).

Veamos el siguiente ejemplo:

Reunir todas las competencias que requiere el estilo discursivo sobresaliente, y más aún las específicas necesarias para escribir y preparar textos que van a editarse y publicarse, no es algo muy improbable, pero sí muy difícil. El editor que se tope con un autor con una competencia completa, sin fisuras, deficiencias o momentos bajos, puede considerarse muy afortunado, entre otras cosas, por el trabajo y el dinero que le ahorrará. Pero por mucho que sueñe con ello, lo cierto es que, aun revisando y preparando el autor (escritor o traductor) su obra o versión en lo posible, esta va a revelar fallos y carencias que no trascenderán al impreso sólo si el editor apuntala la labor del autor mediante pautas y trabajos de remodelación concretos.

Veamos ahora la versión normalizada:

Reunir todas las competencias que requiere el estilo discursivo sobresaliente $-\mathrm{y}$ más aún, aquellas específicas que son necesarias para escribir y preparar textos que van a editarse y publicarse- es algo probable, pero muy difícil. El editor que se tope con un autor con una competencia completa, sin fisuras o deficiencias, puede considerarse muy afortunado (entre otras cosas, por el trabajo y el dinero que le ahorrará). Pero por mucho que sueñe con ello, lo cierto es que, aun cuando el autor (escritor o traductor) haya

\footnotetext{
† A propósito de este tema, Graciela Reyes (1988: 48-49) retoma la anécdota del filólogo Dámaso Alonso, quien en su artículo «Límites de la estilística» cuenta el error dialectal de un ilustre hispanista extranjero que, para demostrar su dominio del español castizo, comenzó su exposición en un congreso internacional con estas palabras: «Yo inclino mi cholla (cabeza) ante esas banderas...».

* Páginas de Guarda. Revista de Lenguaje, Edición y Cultura Escrita. <www.paginasdeguarda.com.ar>.
} 
revisado y preparado su obra o versión lo mejor posible, de todos modos va a haber fallos y carencias que luego no trascenderán al impreso solo si el editor apuntala la labor del autor mediante pautas y trabajos de remodelación concretos.

En este fragmento se han aplicado los siguientes procedimientos de corrección:

\section{Modulación}

Consiste en introducir cambios que no varían la estructura del sintagma, pero la completan o mejoran en algún sentido para facilitar su legibilidad. Por ejemplo, la referencia anafórica establecida en el original por el artículo definido las, es reemplazada por el pronombre demostrativo aquellas que, sumada a la permutación de la proposición adjetiva que son necesarias en lugar del adjetivo necesarias, da como resultado un estilo más cohesivo y, por lo tanto, de más fácil comprensión.

\section{[...] las específicas necesarias para escribir [...] \\ [...] aquellas que son específicas para escribir [...]}

Un ejemplo de modulación en el nivel ortotipográfico es el reemplazo de la raya corta o del paréntesis en lugar de las comas para señalar la estructura parentética $\mathrm{Si}$ comparamos ambas versiones, veremos que en la segunda, la información se muestra más jerarquizada, por lo que es más fácil comprender las relaciones de sentido dentro del párrafo.

Reunir todas las competencias que requiere el estilo discursivo sobresaliente, y más aún las específicas necesarias para escribir y preparar textos que van a editarse y publicarse, no es algo muy improbable, pero sí muy difícil. El editor que se tope con un autor con una competencia completa, sin fisuras, deficiencias o momentos bajos, puede considerarse muy afortunado, entre otras cosas, por el trabajo y el dinero que le ahorrará.

Reunir todas las competencias que requiere el estilo discursivo sobresaliente $-y$ más aún, aquellas específicas que son necesarias para escribir y preparar textos que van a editarse y publicarse - es algo probable, pero muy difícil. El editor que se tope con un autor con una competencia completa, sin fisuras o deficiencias, puede considerarse muy afortunado (entre otras cosas, por el trabajo y el dinero que le ahorrará). 


\section{Transposición}

Consiste en la modificación de la categoría gramatical de una parte de la oración sin alterar su sentido. En nuestro ejemplo, se han reemplazado los dos gerundios, revisando y preparando, por las formas verbales del pretérito perfecto del subjuntivo haya revisado y preparado. Además, se ha modificado la estructura de la proposición concesiva de [...] lo cierto es que... esta va a revelar... en [...] lo cierto es que aun cuando... de todos modos...

[...] lo cierto es que, aun revisando y preparando el autor (escritor o traductor) su obra o versión en lo posible, esta va a revelar fallos y carencias que no trascenderán al impreso [...]

[...] lo cierto es que, aun cuando el autor (escritor o traductor) haya revisado y preparado su obra o versión lo mejor posible, de todos modos va a haber fallos y carencias que no trascenderán al impreso [...]

Otra forma de la transposición es el hipérbaton (de hecho esta palabra proviene del griego íđźs $\beta \alpha \tau o v$, que significa precisamente 'transposición'). El hipérbaton es la figura retórica que consiste en alterar el orden gramatical (para el español [sujeto + verbo + complemento] y dentro del sintagma [elemento rector + elemento regido]) con algún fin poético o comunicativo. En nuestro ejemplo, el sujeto de la oración aparece pospuesto a los gerundios, y el complemento en lo posible resulta ambiguo, puesto que no se sabe si es revisando y preparando en lo posible su obra o, como se resuelve en la corrección, se refiere a la obra o versión, es decir, preparar su obra o versión lo mejor posible.

\section{Transformación}

Consiste en transformar, por ejemplo, una negación en una afirmación o una voz activa en una pasiva o viceversa. 
- Negación:

Como podemos ver en el siguiente ejemplo, dos negaciones juntas, una sintáctica no es y otra morfológica improbable, complican innecesariamente la comprensión, por lo tanto, es conveniente transformarla en una afirmación:

Reunir todas las competencias que requiere el estilo discursivo sobresaliente [...] no es algo muy improbable, pero sí muy difícil.

Reunir todas las competencias que requiere el estilo discursivo sobresaliente [...] es algo probable, pero muy difícil.

- Voz activa en voz pasiva

[...] Hay sustantivos variados: «curre» o «curro», que aunque el DRAE los considera sinónimos, puedo concluir de mensajes orales y de Mensaka que se está desarrollando un pequeño matiz diferenciador.

[...] Hay sustantivos variados: «curre» o «curro» que, aunque son considerados sinónimos por el DRAE, pareciera, a partir del análisis de mensajes orales y de la obra Mensaka, que poseen un pequeño matiz diferenciador.

- Reemplazo de formas léxicas usuales por terminología:

[...] En un artículo de La Recherche, este concepto es representado mediante la figura de un hombre que, como una marioneta humana, se encuentra suspendido por hilos de las agujas de distintos relojes. El dibujo sintetiza, de este modo, la idea de que el organismo reacciona de diferente manera según la hora en que es administrado un medicamento.

[...] En un artículo de La Recherche, este concepto es simbolizado mediante la figura de un hombre que, como una marioneta humana, se encuentra suspendido por medio de hilos, de las agujas de distintos relojes. El dibujo sintetiza, de este modo, la idea de que el organismo reacciona de diferente manera según la hora en que le es administrado un medicamento. 


\subsection{La normalización de erratas}

Como señalé antes, la normalización también alcanza a la corrección de pruebas, que consiste no solo en cotejar que los cambios señalados en la corrección de estilo hayan sido incorporados, sino también en identificar y corregir todas las erratas emanadas del proceso mismo de la composición digital.

Para el primer tipo de verificación, como es sabido, es necesario contraponer el archivo pdf con el original en Word y, de esa manera, asegurarse de que no haya ninguna parte del documento plantado que esté ausente o haya sido modificada. Para la segunda, deben corregirse las ditografías (erratas por adición repetitiva de sílabas o de palabras), las haplografías (omisión de letras, sílabas o segmentos textuales), las metátesis (alteración de letras) o la sustitución, letras griegas, símbolos o palabras en otro idioma que el programa del diseñador no reconoce y que, entonces, en las pruebas aparecen tergiversados $\$$.

Un ejemplo de omisión de letras es la errata no corregida en la página de créditos del número inaugural de Páginas de Guarda (2006), en la cual, increíblemente, el nombre de la correctora Laura Villaveirán apareció bajo el título: *Correción.

$\S$ Los errores de la composición digital suelen ser los mismos que cometían los copistas en las cuatro etapas u operaciones básicas de copiado que señala José Luis Moure (2006: 15): «la lectura de un fragmento (o pericopa) del modelo, su memorización, el dictado que de la pericopa el copista se hace a sí mismo, la transcripción y el regreso al modelo. Cada una de ellas puede albergar un traspié específico -de lectura, de memorización, de dictado interior, de ejecución manual o de reinserción visual en el modelo- y dar lugar a la comisión de errores por adición, por omisión, por alteración del orden y por sustitución». 


\section{Errata en la película}

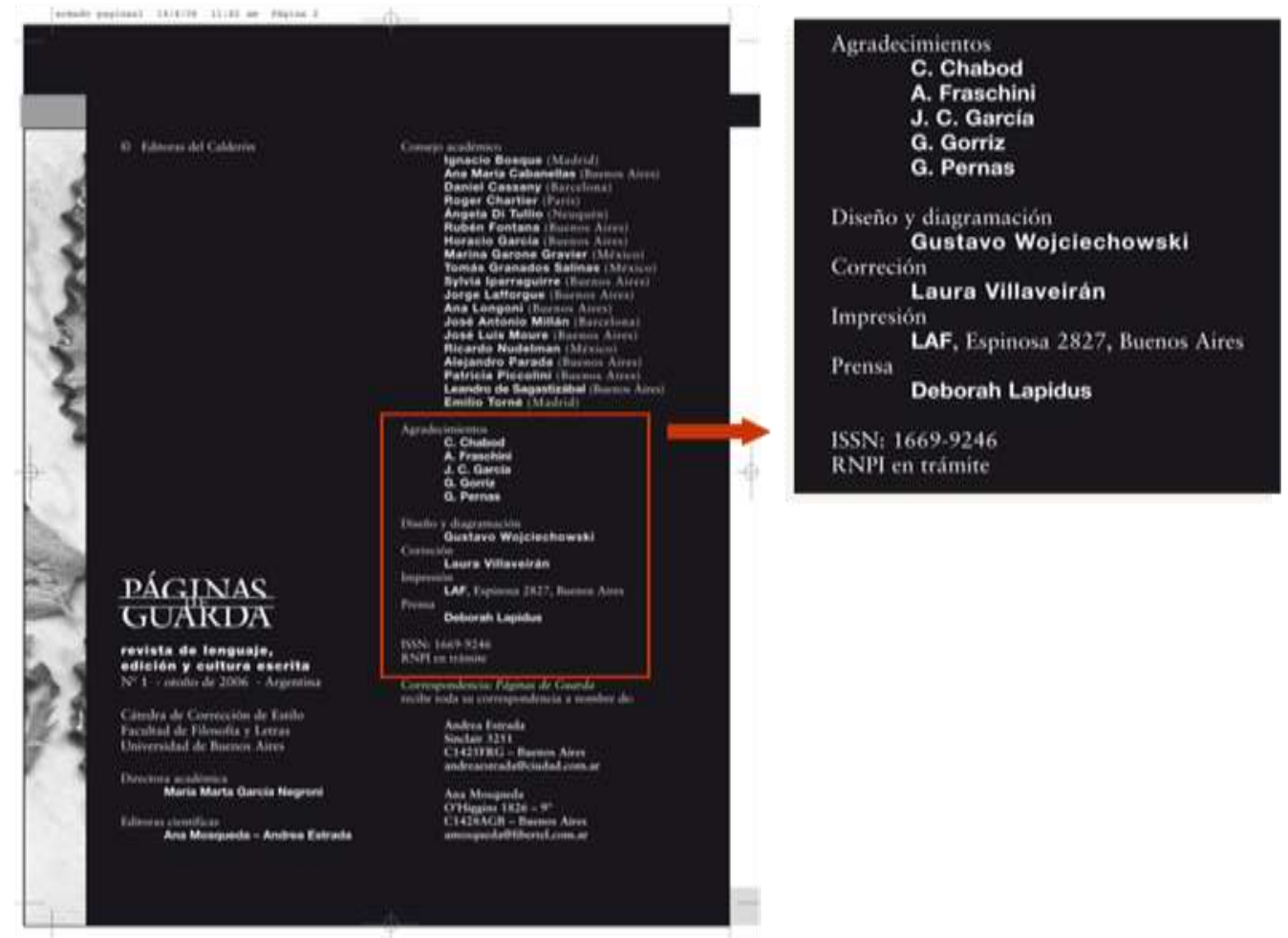

Otro ejemplo significativo de sustitución es la desaparición de todos los signos de euros de la página de suscripciones del volumen 5 (2008) que evidentemente el programa utilizado por el diseñador no reconoció. 


\section{Errata: supresión del signo de euro}

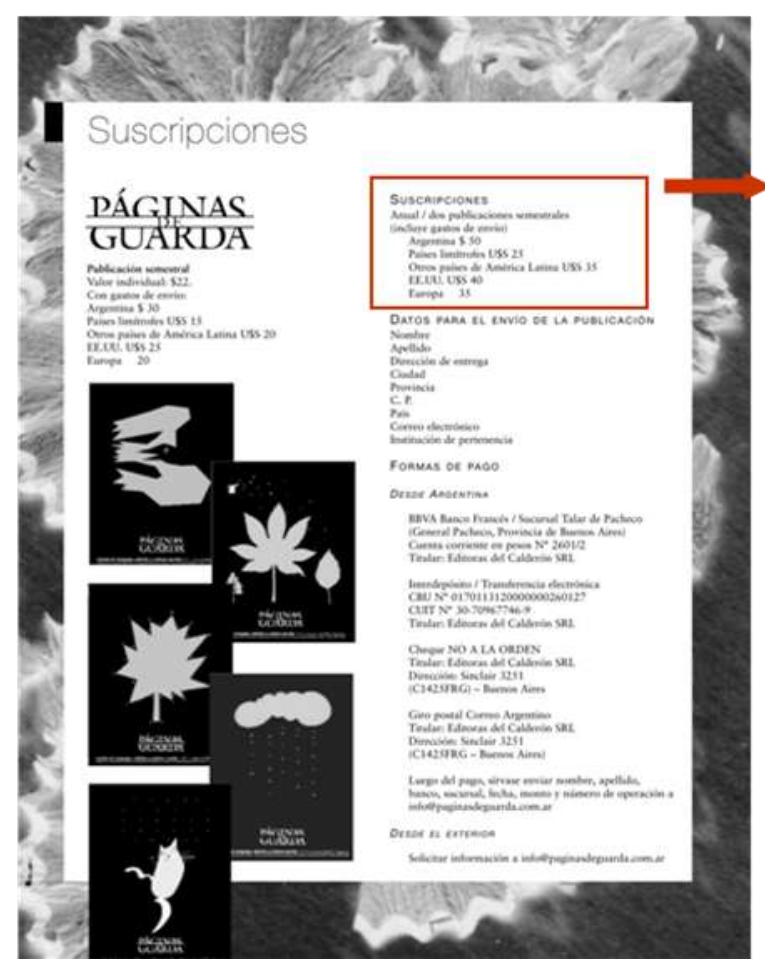

SUSCRIPCIONES

Anual / dos publicaciones semestrales

(incluye gastos de envío)

Argentina $\$ 50$

Paises limitrofes U\$S 25

Otros países de América Latina USS 35

EE.UU. USS 40

Europa 35

Finalmente, en los números 9 y 10, el índice no coincide con la numeración de las distintas secciones, grave errata que descubrió el imprentero por pura casualidad en el número 10 y que, como ya estaban bajadas las películas, resultó imposible de enmendar porque hubiera significado una gran pérdida de tiempo y de dinero.

Otro tipo de erratas que deben ser evitadas son, tal como señala Martínez de Sousa, las referidas a la diacrisis tipográfica, los cortes de palabras y guiones, las líneas viudas y huérfanas de los párrafos, la distribución de los espacios que pueden crear efectos no deseados como las calles, las escaleras o los corrales, entre otros muchos.

En cuanto a la sangría, este gran maestro de la bibliología y la ortotipografía considera que el primer párrafo debe sangrarse, afirmación que sostiene precisamente en un artículo que formó parte del volumen n. ${ }^{\circ} 7$ de Páginas de Guarda. Pero sucede que para las normas editoriales fijadas en la hoja de estilo de esta publicación, el blanco que precede a un título, subtítulo, cuadro o ilustración es de por sí un diacrítico suficiente, como para señalarlo nuevamente con una sangría. 
La tensión entre la necesidad de normalizar las sangrías de todos los artículos que formaban parte de ese volumen y la imposibilidad de, por razones obvias, hacerlo nos llevó a agregar una Nota de la Editora en la cual se aclaraba que, en la diagramación del artículo, se respetaba el criterio de su autor y no el de la publicación. Pero en un aspecto, no normalizado en Páginas de Guarda, tiene razón el gran maestro, puesto que, en el caso de que el párrafo comience en una nueva página, también allí deberá suprimirse la sangría puesto que sería una marca redundante. Como puede verse, este aspecto no está normalizado en Páginas de Guarda.

\section{Normalización de erratas: sangrías y corte de palabras}

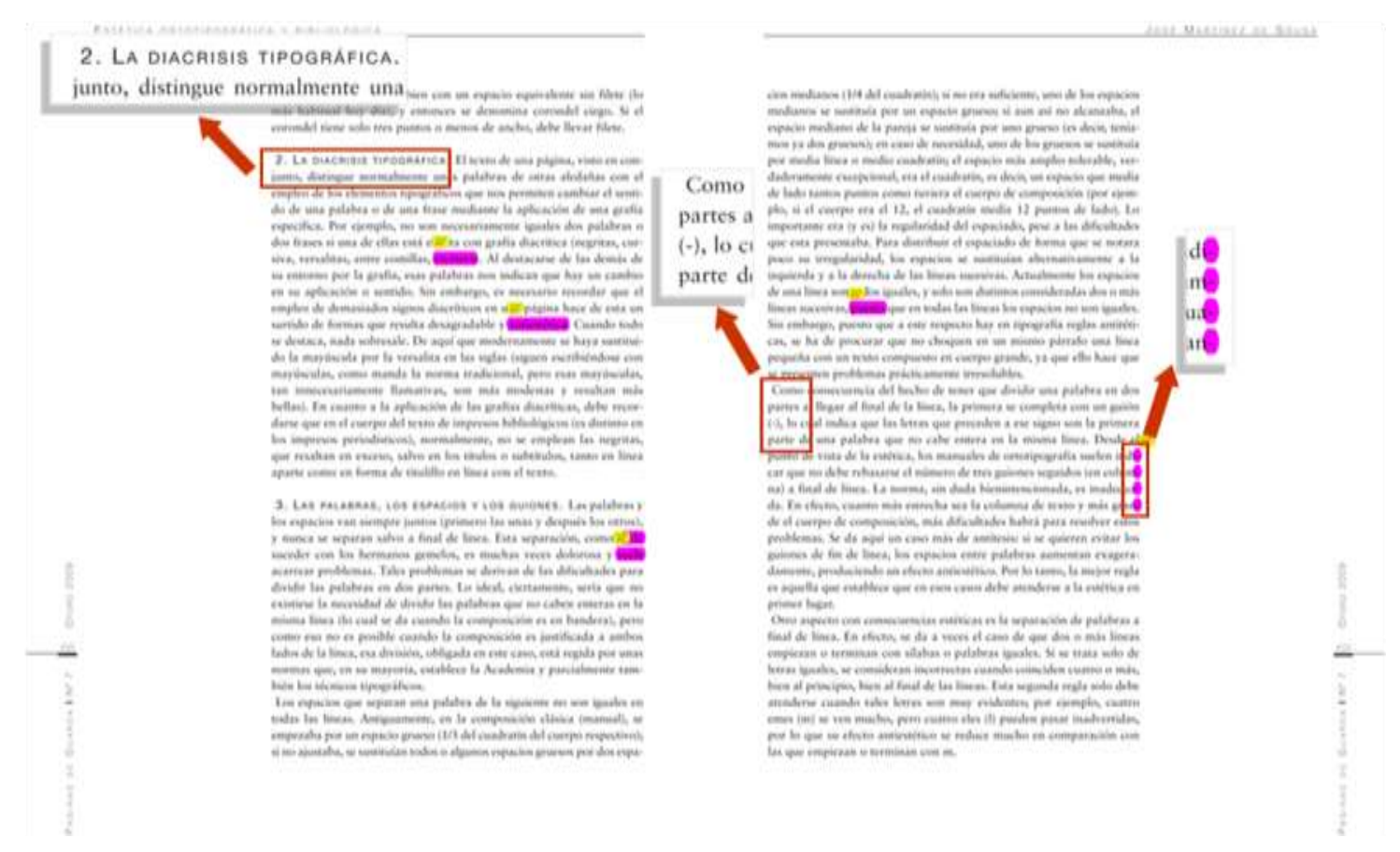

El tamaño de las sangría es otro aspecto a considerar y, como puede verse, en el caso del artículo de Martínez de Sousa, también se respetó a pesar de ser notoriamente menor que la del resto de los artículos de la publicación. 


\section{A MODO DE CONCLUSIÓN}

Como he intentado demostrar a lo largo de este artículo, errores y erratas tienen el mismo protagonismo en la corrección de textos académicos, dado que los criterios y procedimientos de normalización, fundamentales en este tipo de publicaciones, alcanzan tanto a la corrección de estilo como a la corrección de pruebas.

En cuanto a quién corrige los errores y quién las erratas, existen diferentes posturas. Muchos afirman que la persona que corrigió el original de autor debe ser la misma que corrija las primeras pruebas, puesto que al conocer el texto, identificará con mayor facilidad cualquier alteración o faltante en el nuevo documento. Pero también suele decirse que, si los que abordan las distintas instancias de la corrección son personas físicas diferentes, es posible protegerse de dos tipos de efectos nefastos: el de «saturación», que por un exagerado contacto con el texto impide detectar los errores y las erratas más visibles, y el llamado efecto de «omnipotencia», por el cual terminamos creyendo falazmente que corregir errores ajenos nos protege de alguna manera de cometer los propios.

\section{REFERENCIAS BIBLIOGRÁFICAS}

BAJTín, Mijaíl. (1979) [2002]: Estética de la creación verbal, Buenos Aires, Siglo XXI.

BiLlingham, Joe (2007): Edición y corrección de textos, Buenos Aires, FCE (traducción: L. Pérgola).

BOzETTI, Mariana (2010): «Un delicado equilibrio: la corrección literaria», ponencia presentada en las Segundas Jornadas Internacionales sobre la Lengua Española, Buenos Aires, Fundación Litterae y Universidad de Belgrano.

García Negroni, María Marta y Andrea Estrada (2006): «Corrector o corruptor. Saberes y competencias del corrector de estilo», Páginas de Guarda, 1, 2640 .

Hyland, Ken (2000): Disciplinary Discourses, Harlow / New York, Longman.

MARTínez DE SousA, José (2009): «Estética ortotipográfica y bibliológica», Páginas de Guarda, 7, 69-77.

Moure, José Luis (2006): «Errores deseables y erratas cohonestadas», Páginas de Guarda, 1, 11-25. 
PICCOLINI, Patricia (2006): «La selección de originales en la edición universitaria», Páginas de Guarda, 1, 57-66.

REYES, Graciela (1998): Cómo escribir bien en español, Madrid, Arco Libro.

SENZ BUENO, Silvia (2006): «La edición impresa, una cuestión de estilo», Páginas de Guarda, 2, 80-95.

ZORRILla, Alicia María (2004): Normativa lingüística española y corrección de textos, Buenos Aires, Fundación Instituto Superior de Estudios Lingüísticos y Literarios, Litterae. 
